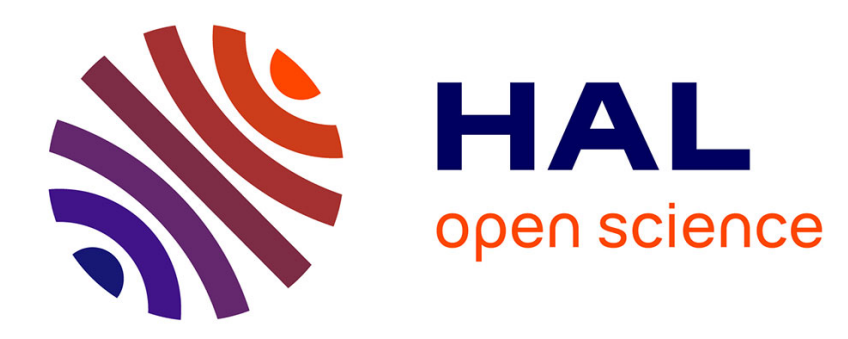

\title{
Multidimensional inequalities and generalized quantile functions
}

\author{
Sinem Bas, Philippe Bich, Alain Chateauneuf
}

\section{To cite this version:}

Sinem Bas, Philippe Bich, Alain Chateauneuf. Multidimensional inequalities and generalized quantile functions. 2016. hal-01313118

\section{HAL Id: hal-01313118 \\ https://hal.science/hal-01313118}

Preprint submitted on 9 May 2016

HAL is a multi-disciplinary open access archive for the deposit and dissemination of scientific research documents, whether they are published or not. The documents may come from teaching and research institutions in France or abroad, or from public or private research centers.
L'archive ouverte pluridisciplinaire HAL, est destinée au dépôt et à la diffusion de documents scientifiques de niveau recherche, publiés ou non, émanant des établissements d'enseignement et de recherche français ou étrangers, des laboratoires publics ou privés. 


\title{
Multidimensional inequalities and generalized quantile functions*
}

\author{
Sinem Bas ${ }^{\dagger} \quad$ Philippe Bich ${ }^{\ddagger} \quad$ Alain Chateauneuf ${ }^{\S}$
}

\begin{abstract}
In this paper, we extend the generalized Yaari dual theory for multidimensional distributions, in the vein of Galichon and Henry's paper [6]. We show how a class of generalized quantiles -which encompasses Galichon and Henry's one or multivariate quantile transform [7] [4] [9]- allows to derive a general representation theorem. Moreover, we derive from this representation theorem a formula which could be applicable to multidimensional measure of inequality.
\end{abstract}

Keywords: multidimensional distributions, quantile, inequality, optimal coupling

*This paper forms part of the research project "The Multiple dimensions of Inequality" (Contract No. ANR 2010 BLANC 1808) of the French National Agency for Research, whose financial support is gratefully acknowledged.

${ }^{\dagger}$ Louvain University, Belgium

${ }^{\ddagger}$ Paris School of Economics, Centre d’Economie de la Sorbonne UMR 8174, Université Paris I Panthéon/ Sorbonne, 106/112 Boulevard de l'Hôpital 75647 Paris Cedex 13. E-mail : bich@univparis1.fr

${ }^{\S}$ IPAG Business School and PSE, Centre d'Economie de la Sorbonne UMR 8174, Université Paris I Panthéon/ Sorbonne, 106/112 Boulevard de l'Hôpital 75647 Paris Cedex 13. E-mail : bich@univparis1.fr 


\section{Introduction}

In a recent paper, Galichon and Henry [6] generalize Yaari dual theory to multidimensional distributions, using Optimal coupling theory. They prove that the preference relationship of a decision maker confronted to choices on multidimensional prospects can be evaluated with a weighted sum of multidimensional quantiles, when this preference relationship can be written as a mutidimensional index that preserves some first order stochastic dominance and satisfies some comonotonic independence property.

The work of Galichon et al. rests on the notion of multidimensional quantile they introduce, $\mu$-quantile. Yet, the choice of a particular multidimensional quantile is not completely obvious or natural, and other multidimensional quantiles have been proposed in the literature, for example multivariate quantile transform (see [7], [4], [9] or [8] for a brief presentation of this notion)

In this paper, we propose an extension of the generalized Yaari dual theory for multidimensional distributions, in the vein of Galichon and Henry's paper. But the class of quantiles we consider encompasses Galichon et al.'s $\mu$-quantile or multivariate quantile transform. In particular, our class of quantiles is not built as the solution of an optimal coupling problem, as in [6], but is required to satisfy natural and simple properties. Also, we avoid to assume some Frechet derivability assumption on the functional which represents the preference of the policymaker, but we try to propose more standard and interpretable assumptions. Third, we try to get some explicit formula of this inequality index, which could be implemented by a policymaker.

The paper is organized as follows. Section 2 recalls the Yaari dual theory of choice for unidimensional distributions. Section 3 introduces our specific framework of multidimensional distributions, offers a general definition of quantiles and states a first representation result. In Section 4 we compare more specifically our results with Galichon et al. ones. In Section 5, it is shown how all the involved parameters can be elicited by a policymaker. Finally, in Section 6, - discussion and concluding remarkssome limitation of the proposed measure is raised, and one suggests a possible route to explore in future work in order to amend it. 


\section{Yaari dual theory of choice for unidimensional distributions}

In this section, we consider $(S, \mathcal{F}, P)$ to be the probability space defined by $S=$ $[0,1], P$ being the Lebesgue measure and $\mathcal{F}$ the $\sigma$-algebra of borelian subsets of $S$. Let $V$ be the set of random variables on $(S, \mathcal{F}, P)$ and $V_{2}$ be the set of elements in $V$ with a finite second moment. Two elements $(X, Y) \in V \times V$ are said to be equal in law (denoted $X={ }_{d} Y$ ) if the probability law of $X$ and $Y$ coincide. For every borelian subset $E$ of $S, \mathbb{I}_{E}$ denotes the characteristic function of $E$, that is $\mathbb{I}_{E}(s)=1$ if $s \in E$ and $\mathbb{I}_{E}(s)=0$ otherwise. For every random variable $X \in V$, we can define its cumulative distribution function $F_{X}(x)=P(X \leq x)$, and its unidimensional quantile $F_{X}^{-1}$, as follows:

$$
F_{X}^{-1}(p)=\inf \{x \in \mathbb{R}: P(X \leq x) \geq p\}
$$

The quantile function can be seen also as an element of $V$ (since $F_{X}^{-1}$, is a measurable real function on $S$ ). Importantly, it is the solution of some optimization problem, called optimal coupling problem, which we now recall.

For every $U \in V_{2}$, define the maximum correlation functional associated to $U$ as follows:

$$
\forall X \in V_{2}, \rho_{U}(X):=\sup _{\tilde{X} \in V_{2}, \tilde{X}=_{d} X} \int_{[0,1]} U(p) \cdot \tilde{X}(p) d p
$$

Choosing $U(p)=p$ (which we now assume in this section), then Hardy-Littlewood inequality guarantees that $F_{X}^{-1}$, is the unique (almost surely) solution $\tilde{X}$ in $V_{2}$ of the above optimization problem. In particular, $\rho_{U}(X):=E\left(F_{X}^{-1}(p) \cdot p\right)$, and it follows that the quantile posses the following well known properties:

Proposition 2.1. (i) For every $X, Y \in V_{2}$ such that $X={ }_{d} Y, F_{X}^{-1}=F_{Y}^{-1}$ almost surely, and $F_{X}^{-1}={ }_{d} X$.

(ii) For every $X, Y \in V_{2}$ and $\lambda \geq 0, F_{X}^{-1}+F_{Y}^{-1}$ and $\lambda F_{X}^{-1}$ are themselves the quantiles of $F_{X}^{-1}+F_{Y}^{-1}$ and of $F_{\lambda X}^{-1}$.

(iii) For every $\lambda \in \mathbf{R}$ and $X$ in $V_{2}$, the quantile of $X+\lambda$ is $F_{X}^{-1}+\lambda$ (almost surely). Proof. Property (i) is a consequence of the definition of the quantile $Q_{X}$ in terms of optimal coupling solution. For property (ii), recall that the solution $F_{X}^{-1} \in V_{2}$ is 
characterized (1) $F_{X}^{-1}$ is increasing $(2) F_{X}^{-1}={ }_{d} X$. Indeed, the first condition can be written: $G(t)=\int_{0}^{t} F_{X}^{-1}$ is continuous and convex, and condition (2) is $\nabla G={ }_{d} X$. Then, from optimal coupling solution, these two conditions characterized the (unique almost surely) solution $F_{X}^{-1}$ (see appendix 7.1.1). Now, let $X^{\prime}=F_{X}^{-1}$ and $Y^{\prime}=F_{Y}^{-1}$. From above, the quantile of $X^{\prime}+Y^{\prime}$ is characterized by (1) $F_{X^{\prime}+Y^{\prime}}^{-1}$ is increasing (2) $F_{X^{\prime}+Y^{\prime}}^{-1}={ }_{d} X^{\prime}+Y^{\prime}$. Thus, $F_{X^{\prime}+Y^{\prime}}^{-1}=F_{X}^{-1}+F_{Y}^{-1}$, because it satisfies the two conditions (1) and (2). This is similar for $F_{\lambda F_{X}^{-1}}^{-1}=\lambda F_{X}^{-1}$. The last property (iii) is a straighforward consequence of the definition of quantile.

In the next section, we shall define a generalized class of multidimensional quantiles using the same requirements.

Now, to state Yaari dual choice theory main representation result, we need to recall comonotonicity concept:

Definition 2.2. Two random variables $X$ and $Y$ in $V$ are comonotonic if

$$
(X(s)-X(t))(Y(s)-Y(t)) \geq 0
$$

almost surely in $(s, t) \in S \times S$.

Comonotonicity can be characterized using maximal correlation functional as follows [2]:

Proposition 2.3. Two random variables $X$ and $Y$ in $V_{2}$ are comonotonic if and only if

$$
\rho_{U}(X+Y)=\rho_{U}(X)+\rho_{U}(Y) .
$$

Then we can state:

Theorem 2.1. (Yaari main representation theorem)

Let $I: V_{2} \rightarrow \mathbf{R}$. The two assertions below are equivalent:

(1) The functional I satisfies:

1. (Normalization) $I\left(\mathbb{1}_{S}\right)=1$

2. (Anonymity) for every $(X, Y) \in V_{2} \times V_{2}, X={ }_{d} Y \Rightarrow I(X)=I(Y)$. 
3. (Inequality Aversion) for every $(X, Y) \in V_{2} \times V_{2}, I(X+Y) \geq I(X)+I(Y)$.

4. (Additive comonotonicity) for every $(X, Y) \in V_{2} \times V_{2}$ comonotonic, $I(X+Y)=$ $I(X)+I(Y)$.

(2) There exists a unique convex and non-decreasing function $f:[0,1] \rightarrow[0,1]$, $f(0)=0, f(1)=1$ such that:

$$
I(X)=\int_{0}^{1} f\left(1-F_{X}(p)\right) d p=\int_{0}^{1} F_{X}^{-1},(p) \cdot f^{\prime}(1-p) d p=\int_{S} X d(f \circ P)
$$

where $\int_{S} X d(f \circ P)$ is the Choquet integral of $X$ with respect to capacity $f \circ P$.

In the theorem above, we recall that $f \circ P$ is defined on the set of borelian subsets of $S$ as follows: for every borelian $E \subset S, \phi \circ P(E)=f(P(E))$. The function obtained is no longer a probability (it is not additive in general), but it is a capacity, and Choquet integral is a way to extend the standard expectation with respect to a probability to the case where the probability is replaced by a capacity.

\section{A first representation result in the multidimen- sional case}

\subsection{Framework}

In this section, we consider a more general framework than in the previous section. Let $(S, \mathcal{F}, P)$ be a probability space. For every subset $A$ of $\mathbb{R}^{n}$, denote $\mathcal{B}_{A}$ the Borelian $\sigma$-algebra on $A$. Let $E($.$) be the expectation operator with respect to the probability$ $P$. Let $V$ be the set of random $n$-dimensional vectors on $(S, \mathcal{F}, P)$, i.e. $V=\{X$ : $(S, \mathcal{F}, P) \rightarrow\left(\mathbb{R}^{n}, \mathcal{B}_{\mathbb{R}^{n}}\right)$ measurable $\}$. For every $X \in V$, we write $X=\left(X_{1}, \ldots, X_{n}\right)$, where for every $s \in S$, we define $X(s)=\left(X_{1}(s), \ldots, X_{i}(s), \ldots, X_{n}(s)\right)$. The probability distribution of $X$ on $\left(\mathbb{R}^{n}, \mathcal{B}_{\mathbb{R}^{n}}\right)$ is denoted $P^{X}$. We let $V_{2}$ be the set of elements in $V$ with finite second moments, i.e. $V_{2}=\left\{X \in V: \forall i \in\{1,2, \ldots, n\}, E\left(X_{i}^{2}\right)<+\infty\right\}$. For every $X$ and $Y$ in $V$, we denote $X={ }_{d} Y$ if $X$ and $Y$ have the same distribution, that is $P^{X}=P^{Y}$. Recall the definition of the standard scalar product $<.,>$ on $V_{2}$ : for every $X=\left(X_{1}, \ldots, X_{n}\right)$ and $Y=\left(Y_{1}, \ldots, Y_{n}\right),\langle X, Y\rangle=\sum_{i=1}^{n} E\left(X_{i} Y_{i}\right)=$ $\sum_{i=1}^{n} \int_{S} X_{i}(s) Y_{i}(s) d P(s)$. Hereafter, $X . Y$ simply denotes $\sum_{i=1}^{n} X_{i} . Y_{i}$. 


\subsection{A new definition of quantiles}

We now propose a new definition of multidimensional quantile through some natural properties that are satisfied in the unidimensional case. Importantly, we allow the quantile operator to be defined on a strict subset $V_{2}^{\prime}$ of the set of $n$-random variables $V_{2}$ ( $V_{2}^{\prime}$ will often be chosen equal to $V_{2}$, but we will also sometimes consider $V_{2}^{\prime}$ to be, for example, the set of comonotonic or anti-comonotonic (when $n=2$ ) random vectors.) In the following, for every sets $E$ and $F, \mathcal{F}(E, F)$ denote the set of functions from $E$ to $F$.

Definition 3.1. Consider a n-dimensional random variable $U \in V_{2}$ with values in $[0,1]^{n}$. Let $V_{2}^{\prime}$ be a convex cone of $V_{2}$ containing the constant random variables. An operator $Q: V_{2}^{\prime} \rightarrow \mathcal{F}\left([0,1]^{n}, \mathbf{R}^{n}\right)$ is a $U$-quantile operator (or simply of quantile operator when $U$ is implicit) if it satisfies the three following properties: ${ }^{1}$

1. (Law of quantiles) For every $(X, Y) \in V_{2}^{\prime} \times V_{2}^{\prime}$ such that $X={ }_{d} Y, Q_{X}=Q_{Y}$ almost surely, and $Q_{X}(U)={ }_{d} X$.

2. (Sum of quantiles) For every $\lambda \geq 0$ and $(X, Y) \in V_{2}^{\prime} \times V_{2}^{\prime}, Q_{X}+Q_{Y}$ and $Q_{\lambda X}$ are the quantiles of some random variable in $V_{2}^{\prime}$.

3. For every $\lambda \in \mathbf{R}$ and $X$ in $V_{2}^{\prime}$, the quantile of $X+\lambda\left(\mathbb{1}_{S}, \ldots, \mathbb{1}_{S}\right)$ is $Q_{X}+\lambda$. Sometimes we will assume one of the three additional assumptions:

4. For every strictly positive reals $\lambda_{1}, \ldots, \lambda_{n}$, the function $p \in \mathbf{R}^{n} \rightarrow\left(\lambda_{1} p_{1}, \ldots, \lambda_{n} p_{n}\right)$ is the quantile of some random variable in $V_{2}^{\prime}$.

5. (when $n \geq 2$ ) For every symmetric definite positive $n$-matrix $A$, the function $p \rightarrow A p$ is the quantile of some random variable in $V_{2}^{\prime}$.

6. If $X=\left(X_{1}, \ldots, X_{n}\right) \in V_{2}^{\prime}$, where the $X_{i}$ 's are mutually independent, then one has $Q_{X}(p)=\left(Q_{X_{1}}\left(p_{1}\right), \ldots, Q_{X_{i}}\left(p_{i}\right), \ldots, Q_{X_{n}}\left(p_{n}\right)\right)$ for every $p=\left(p_{1}, \ldots, p_{i}, \ldots, p_{n}\right) \in$ $[0,1]^{n}$, where each $Q_{X_{i}}$ is the one-dimensional quantile of $X_{i}$.

Remark 3.1. For $n=1$, the standard unidimensional quantile defines a quantile operator by $X \in V_{2} \rightarrow Q_{X}=F_{X}^{-1} \in \mathcal{F}([0,1], \mathbf{R})$ (see Proposition 2.1). Conversely, if

1. Hereafter, $Q(X)$ is also denoted $Q_{X}$, and is called a quantile. Sometimes, to make more explicit the parameter $U$, we will call $Q_{X}$ a $U$-quantile, and will denote it $Q_{X}^{U}$. 
Property 6 is assumed to be true, then for $n=1$, any quantile operator coincides with the standard unidimensional quantile.

In the three next subsections, we give particular examples of quantile operators illustrating Definition 3.1.

\subsection{Example 1: multidimensional quantile on the set of comono- tonic vectors}

Consider $V_{2}^{\prime}$ the class of comonotonic random vectors $X=\left(X_{1}, \ldots, X_{n}\right) \in V_{2}$, in the sense that for every $(i, j) \in\{1, \ldots, n\}^{2},\left(X_{i}, X_{j}\right)$ is comonotonic. Fix $U$ a random vector whose components are independent and uniformly distributed with values in $[0,1]$. Then a quantile operator can be defined by

$$
\forall X=\left(X_{1}, \ldots, X_{N}\right) \in V_{2}^{\prime}, Q_{X}\left(p_{1}, \ldots, p_{n}\right)=\left(F_{X_{1}}^{-1}\left(p_{1}\right), \ldots, F_{X_{n}}^{-1}\left(p_{n}\right)\right)
$$

Importantly, this could not define a quantile operator on whole $V_{2}$, because when $X$ is not comonotonic, the law of $Q_{X}(U)$ and the law of $X$ could be different (in particular it does not satisfy the first requirement of quantile operator).

We now check that this operator satisfies the requirements of Definition 3.1. Thus $V_{2}^{\prime}$ is a convex cone and contains the constant random vectors. Second, Point 1 in Definition 3.1 is true, that is for every $(X, Y) \in V_{2}^{\prime} \times V_{2}^{\prime}$ such that $X={ }_{d} Y, Q_{X}=Q_{Y}$ almost surely, and $Q_{X}(U)={ }_{d} X$. The second equality is a consequence of comonotonicity (see [3]: for comonotonic vectors, equality of two random vectors can be checked component by component), and the first one is true because it's true in the unidimensional case. Third, Point 2 in Definition 3.1 is true, because for every $\lambda \geq 0$ and $X, Y$ in $V_{2}^{\prime}, Q_{X}+Q_{Y}=\left(F_{X_{1}}^{-1}+F_{Y_{1}}^{-1}, \ldots, F_{X_{n}}^{-1}+F_{Y_{1}}^{-1}\right)$ and $Q_{\lambda X}=\left(\lambda F_{X_{1}}^{-1}, \ldots, \lambda F_{X_{n}}^{-1}\right)$ are the quantile of $\left(F_{X_{1}}^{-1}(U)+F_{Y_{1}}^{-1}(U), \ldots, F_{X_{n}}^{-1}(U)+F_{Y_{1}}^{-1}(U)\right)$ and of $\left(\lambda F_{X_{1}}^{-1}(U), \ldots, \lambda F_{X_{n}}^{-1}(U)\right)$ (see Proposition 2.1).

Now, the last point is clear, since for every $\lambda \in \mathbf{R}$ and $X$ in $V_{2}^{\prime}$, the quantile of $X+\lambda\left(\mathbb{I}_{S}, \ldots, \mathbb{I}_{S}\right)=\left(X_{1}+\lambda \mathbb{I}_{S}, \ldots, X_{n}+\lambda \mathbb{I}_{S}\right)$ is $\left(F_{X_{1}+\lambda \mathbb{I}_{S}}^{-1}, \ldots, F_{X_{n}+\lambda \mathbb{I}_{S}}^{-1}\right)=Q_{X}+\lambda$. 


\subsection{Example 2: quantile on the set of anti-comonotonic 2- random vectors}

Recall that a 2-dimensional random vector $\left(X_{1}, X_{2}\right)$ is anti-comonotonic if $\left(X_{1},-X_{2}\right)$ is comonotonic. Let $V_{2}^{\prime}$ the class of anti-comonotonic random vectors $X=\left(X_{1}, X_{2}\right) \in$ $V_{2}$ (thus in this subsection, $n=2$ ). A quantile operator can be defined by

$$
\forall X \in V_{2}^{\prime}, Q_{X}\left(p_{1}, p_{2}\right)=\left(F_{X_{1}}^{-1}\left(p_{1}\right), F_{X_{2}}^{-1}\left(1-p_{2}\right)\right)
$$

We let the reader check that it satisfies the requirements of Definition 3.1, which is similar to the previous example.

\subsection{Example 3: Galichon et al. $\mu$-quantile}

Let $U \in V_{2}$ whose probability law $\mu$ has a finite second moment and is absolutely continuous with respect to Lebesgue measure. Assume $U$ takes its values in $[0,1]^{n}$. In this subsection, $V_{2}^{\prime}=V_{2}$. Given $X \in V_{2}$, recall that the pair $(\tilde{X}, U) \in V_{2} \times V_{2}$ is called an optimal coupling if $\tilde{X}$ is a solution of the following optimization problem:

$$
\sup _{\tilde{X}=d_{d} X} E(\tilde{X} \cdot U)
$$

From Optimal coupling theory (see Appendix 7.1.1):

Definition 3.2. (Galichon et al.) For every $X \in V_{2}$, the unique (almost surely) $\nabla f:[0,1]^{n} \rightarrow \mathbf{R}^{n}$ such that $(\nabla f(U), U)$ is an optimal coupling is called the $\mu$-quantile of $X$. We shall denote it $Q_{X}^{\mu}$. If $\mu$ is the probability of some $U \in V_{2}$, we shall say by extension that $Q_{X}^{\mu}$ is the $U$-quantile of $X$.

In particular, the $\mu$-quantile $Q_{X}^{\mu}$ does not depend on $U$, but on the law of $U$.

Proposition 3.3. The $\mu$-quantile of Galichon et al. defines a U-quantile operator on $V_{2}$.

See the proof in Appendix 7.2. 


\subsection{A fourth example: multivariate quantile transform}

The multivariate quantile transform was introduced by [7], [4] or [9]. See also [8] for a brief presentation of this concept. We recall the definition of multivariate quantile transform in the case $n=2$, the general case being a straightforward generalization.

Let $(S, \mathcal{F}, P)$ be any probabilized space. Let $U_{1}, U_{2}$ be two independent and identically $U([0,1])$-distributed random variables on $S$, and $U=\left(U_{1}, U_{2}\right)$. Define a $U$-quantile operator as follows: for every $X=\left(X_{1}, X_{2}\right)$, a random vector on $S$, $Q_{X}=\left(Q_{X}^{1}, Q_{X}^{2}\right)$ is defined by

$$
Q_{X}^{1}\left(p_{1}, p_{2}\right)=F_{X_{1}}^{-1}\left(p_{1}\right)
$$

where $F_{X_{1}}^{-1}$ is the one-dimensional quantile of $X_{1}$, and by

$$
Q_{X}^{2}\left(p_{1}, p_{2}\right)=\inf \left\{x \in \mathbf{R}: P\left(X_{2} \leq x \mid F_{X_{1}}^{-1}\left(U_{1}\right)=F_{X_{1}}^{-1}\left(p_{1}\right)\right) \geq p_{2}\right\},
$$

i.e. $Q_{X}^{2}\left(p_{1}, p_{2}\right)$ is the one-dimensional conditionnal quantile of $X_{2}$ given the first component $Q_{X}^{1}$.

Proposition 3.4. The multivariate quantile transform defines a $U$-quantile operator on $V_{2}$.

See the proof in appendix 7.3.

Interestingly, the multivariate quantile transform is not a particular case of Galichon et al.'s quantile:

Proposition 3.5. Denote by $Q_{X}$ the multivariate quantile of every $X \in V_{2}$. There does not exists some measure $\mu$ such that for every $X \in V_{2}, Q_{X}=Q_{X}^{\mu}$, where $Q^{\mu}$ denotes Galichon et al.'s quantile operator.

Proof. Consider the case $n=2$. Let $U_{1}, U_{2}$ be two independent and identically $U([0,1])$-distributed random variables on $S$. By contradiction, assume that there exists some probability measure $\mu$ such that $Q_{X}=Q_{X}^{\mu}$. In particular, since $Q_{\left(U^{1},-U^{1}\right)}\left(p_{1}, p_{2}\right)=$ $\left(p_{1},-p_{1}\right)$, we should have $Q_{\left(U^{1},-U^{1}\right)}^{\mu}=\left(p_{1},-p_{1}\right)$. But by definition of $Q_{\left(U^{1},-U^{1}\right)}^{\mu}$, there is a convex function $f: \mathbf{R}^{2} \rightarrow \mathbf{R}$ such that $Q_{\left(U^{1},-U^{1}\right)}^{\mu}=\nabla f$. Thus, $\frac{\partial f}{\partial p_{1}}\left(p_{1}, p_{2}\right)=p_{1}$ 
and $\frac{\partial f}{\partial p_{2}}\left(p_{1}, p_{2}\right)=-p_{1}$, which implies that the Hessian of $f$ is equal to $\left(\begin{array}{cc}1 & 0 \\ 0 & -1\end{array}\right)$, a contradiction with the convexity of $f$.

\subsection{The main representation result}

Fix $U \in V_{2}$, and consider $Q$ a $U$-quantile operator on $V_{2}^{\prime}$, a convex cone of $V_{2}$ containing the constant random variables. The preferences of the decision maker on $V_{2}$ are now assumed to be represented by a function $I: V_{2} \rightarrow \mathbf{R}$.

\subsubsection{The main assumptions}

Throughout this paper, we assume that the function $I: V_{2} \rightarrow \mathbf{R}$ satisfies the following assumptions:

\section{Assumptions on $I$}

1. Normalization: $I\left(\mathbb{I}_{S}, \ldots, \mathbb{I}_{S}\right)=1$

2. Monotonicity: $\forall(X, Y) \in V_{2} \times V_{2}, X \geq Y \Rightarrow I(X) \geq I(Y)$.

3. Inequality Aversion: for every $(X, Y) \in V_{2}^{\prime} \times V_{2}^{\prime}$ such that $I(X)=I(Y)$ and every $\lambda \in[0,1]$, we have $I(\lambda X+(1-\lambda) Y) \geq I(X)$.

4. Positive homogeneity: for every $\lambda \geq 0$ and $X \in V_{2}$, we have $I(\lambda X)=\lambda I(X)$.

5. Additivity on Quantiles: for every $(X, Y) \in V_{2}^{\prime} \times V_{2}^{\prime}$, we have $I\left(Q_{X}(U)+\right.$ $\left.Q_{Y}(U)\right)=I\left(Q_{X}(U)\right)+I\left(Q_{Y}(U)\right)$.

6. Neutrality: $I(X)$ only depends of the law of $X$, that is for every $(X, Y) \in V_{2} \times V_{2}$ with the same law, $I(X)=I(Y)$.

Remark 3.2. Inequality aversion is equivalent to concavity, i.e. to: for every $(X, Y) \in$ $V_{2}^{\prime} \times V_{2}^{\prime}$ and $\lambda \in[0,1], I(\lambda X+(1-\lambda) Y) \geq \lambda I(X)+(1-\lambda) I(Y)$. Indeed, concavity clearly implies inequality aversion. Conversely, if inequality aversion is true, consider $X^{\prime}=X-I(X)\left(\mathbb{1}_{S}, \ldots, \mathbb{1}_{S}\right), Y^{\prime}=Y-I(Y)\left(\mathbb{1}_{S}, \ldots, \mathbb{1}_{S}\right)$. From neutrality and from point 3 in Definition 3.1, we get $I\left(X^{\prime}\right)=I\left(Q_{X^{\prime}}(U)\right)=I\left(\left(Q_{X}(U)-I(X)\right)=I\left(Q_{X}(U)\right)-\right.$ $I(X)$ (from additivity on quantiles) which is finally 0 , and similarly $I\left(Y^{\prime}\right)=0$. Then Inequality Aversion at $X^{\prime}$ and $Y^{\prime}$ implies the above inequality. Indeed, first notice that for every constant $c$ and $X \in V_{2}^{\prime}, I(X+c)=I\left(Q_{X}(U)+c\right)($ since $X+c$ and 
$Q_{X}(U)+c$ have the same law from point 3 in Definition 3.1) which is also equal to $I\left(Q_{X}(U)\right)+c=I(X)+c$ from Additivity on Quantiles, thus finally $I(X+c)=I(X)+c$. Now, from Inequality Aversion at $X^{\prime}$ and $Y^{\prime}$ we get $I\left(\lambda X^{\prime}+(1-\lambda) Y^{\prime}\right) \geq 0$ or also $I\left(\lambda X+(1-\lambda) Y-\left(\lambda I(X)\left(\mathbb{1}_{S}, \ldots, \mathbb{1}_{S}\right)+(1-\lambda) I(Y)\left(\mathbb{1}_{S}, \ldots, \mathbb{1}_{S}\right)\right) \geq 0\right.$ and developping and from $I(X+c)=I(X)+c$, we finally get concavity.

\subsubsection{The representation theorem}

The proof of the following theorem can be found in Appendix 7.4.

Theorem 3.3. The mapping $I: V_{2} \rightarrow \mathbf{R}$ satisfies Assumptions 1-6 above if and only if there exists a function $\phi:[0,1]^{n} \rightarrow \mathbf{R}^{n}$ whose components are non negative (almost surely) and such that:

(i) $E\left(\left(\mathbb{1}_{S}, \ldots, \mathbb{1}_{S}\right) \cdot \phi(U)\right)=1$.

(ii) For every $X \in V_{2}^{\prime}, I(X)=\int Q_{X}(U) . \phi(U) d P=\min _{\tilde{X}={ }_{d} X} \int \tilde{X} . \phi(U) d P$, i.e. $I($.$) is the min correlation risk measure with respect to \phi(U)$.

Corollary 3.4. Assume that that the support of $P^{U}$ is $[0,1]^{n}$.

- In the above theorem, $\phi=\nabla g(-i d)$ for some convex function $g$.

- If we additionnaly assume that $Q$ satisfies point 4 in Definition 3.1, then $\phi$ is separable, i.e. $\phi\left(p_{1}, \ldots, p_{n}\right)=\left(\phi_{1}\left(p_{1}\right), \ldots, \phi_{n}\left(p_{n}\right)\right)$, where each $\phi_{i}$ is a decreasing non negative function.

- Last, when $n \geq 2$, if $Q$ also satisfies point 5 in Definition 3.1, then there exists $a \in \mathbf{R}_{+}, b=\left(b_{1}, \ldots, b_{n}\right) \in \mathbf{R}^{n}$, such that $\phi\left(p_{1}, \ldots, p_{n}\right)=\left(b_{1}, \ldots, b_{n}\right)-a \cdot\left(p_{1}, \ldots, p_{n}\right)$. In this case, Condition (iii) above is always satisfied, and can be removed in the equivalence.

Remark 3.5. First, in the two main examples of quantile operators of this paper (Galichon et al $\mu$-quantile and multivariate quantile operator), $V_{2}^{\prime}$ is the total space $V_{2}$.

Second, the interpretation of this representation theorem is similar to Yaari standard interpretation of dual choice theory: the formula $I(X)=\int Q_{X}(U) \cdot \phi(U) d P$ could be seen as a corrected mean of $X$, the correction being a way to compensate the inequality created by the distribution $X$. Indeed, the quantile $Q_{X}$ can be seen as an attempt to 
re-order the distribution $X$ (indeed, for $n=1$, the unidimensional quantile corresponds to standard increasing re-ordering). Then, the weight $\phi(U)$ compensates the effect due to the ordering of $Q_{X}$, which is a consequence of $I(X)=\min _{\tilde{X}={ }_{d} X} \int \tilde{X} \cdot \phi(U) d P$. Indeed, this equality implies that $-Q_{X}(U)$ and $\phi(U)$ are optimally coupled, which corresponds to the intuition that $\phi(U)$ and $-Q_{X}(U)$ are "ordered" in opposite directions. In particular, in the unidimensional case, this implies that $\phi$ is a decreasing function, thus high values of $X$ receive low weights when evaluated by the decision maker.

Recall also that for every $Y \in V_{2}$ with non negative components, the max correlation risk measure $\Psi_{Y}$ is defined by $\Psi_{Y}(X)=\max _{\tilde{X}=_{d} X} \int \tilde{X} . Y d P$. This is a coherent (i.e. monotone, positively homogeneous and subadditive) and law invariant risk measure (see [8], p. 192). In particular it is convex. Thus, in the above theorem, $I(X)=$ $-\Psi_{\phi(U)}(-X)$ is concave.

In the unidimensional case $n=1$, we can choose the quantile operator to be equal to the standard 1-dimensional quantile, i.e. $Q_{X}=F_{X}^{-1}$. Then we get

Corollary 3.6. For $n=1, S=[0,1], U(p)=p$ and $P$ be the Lebesgue measure, Theorem 3.3 is equivalent to Yaari Theorem 2.1.

Proof. Indeed, from corollary above, $\phi(x)=g^{\prime}(-x)$ for some concave function $g$ defined on $[-1,0]$, which can be chosen such that $g(-1)=0$. Since $\phi$ is non negative, $g$ is also non decreasing. Define $f(x)=g(x-1)$ on $[0,1]$. It is convex, non decreasing, $f(0)=0$. Moreover, Theorem 3.3 delivers $I(X)=\int F_{X}^{-1}(p) \cdot g^{\prime}(-p) d p=$ $\int F_{X}^{-1}(p) \cdot f^{\prime}(1-p) d p=\int X(f \circ P)$, and finally $f(1)=1$ is a consequence of normalization assumption $\left(\right.$ since $\left.I(1)=1=\int F_{X}^{-1}(p) \cdot f^{\prime}(1-p) d p=f(1)\right)$.

\section{The case of $\mu$-quantile: Galichon and Henry re- visited}

In this subsection, we compare Galichon et al. Representation Theorem 5 (see [6]) with Theorem 3.3. Let $U \in V_{2}$ whose probability law $\mu$ is absolutely continuous with 
respect to Lebesgue measure. To make the comparison easier, we first recall Axioms 1', 2' and 3' used in Galichon et al. [6].

Remark that without any loss of generality, we can assume that $I$ satisfies the normalization assumption in subsection 3.7.1.

Axiom 1' The functional $I$ is continuous on $V^{2}$, and at least at one point its Frechet derivative exists and is non-zero.

For every $(X, Y) \in V_{2}$, we say that $X \mu$-first order stochastically dominates $Y$ (resp. $X \mu$-first order strictly stochastically dominates $Y$ ) if $Q_{X}(U) \geq Q_{Y}(U)$ almost surely (resp. $Q_{X}(U)>Q_{Y}(U)$ almost surely).

Axiom 2' The functional $I$ preserves $\mu$-first order stochastic dominance, in the sense that if $X \mu$-first order stochastically dominates $Y$, then $I(X) \geq I(Y)$, and if $X \mu$-first order strictly stochastically dominates $Y$, then $I(X)>I(Y)$.

For the last axiom, recall the standard definition of the maximal correlation functional: for every $X \in V_{2}, \rho_{\mu}(X):=\sup _{\tilde{U} \in V_{2}, \tilde{U}={ }_{d} U} \int X . \tilde{U} d P$ The following definition of $\mu$-comonotonicity was introduced by Galichon et al.

Definition 4.1. We say $X_{1}, \ldots, X_{n}$ in $V_{2}$ are $\mu$-comonotonic if

$$
\rho_{\mu}\left(\sum_{i=1}^{n} X_{i}\right)=\sum_{i=1}^{n} \rho_{\mu}\left(X_{i}\right) .
$$

Axiom 3' If $X, Y$ and $Z$ are $\mu$-comonotonic in $V_{2}$, then for every $\alpha \in[0,1], I(X) \geq$ $I(Y)$ implies

$$
I(\alpha X+(1-\alpha) Z) \geq I(\alpha Y+(1-\alpha) Z)
$$

Theorem 4.1. (Galichon-Henry [6]) The functional I satisfies Axioms 1', 2', 3' and Inequality aversion if and only there exists a functional $I^{\prime}: V_{2} \rightarrow \mathbf{R}$ representing the same preference relation on $V_{2}$ (in the sense $I(X) \leq I(Y)$ if and only if $I^{\prime}(X) \leq$ $\left.I^{\prime}(Y)\right)$ which satisfies the 6 Assumptions in subsection 3.7.1. From Theorem 3.3, this 
is equivalent to the existence $a \in \mathbf{R}, b=\left(b_{1}, \ldots, b_{n}\right) \in \mathbf{R}^{n}$, such that $\phi\left(p_{1}, \ldots, p_{n}\right)=$ $\left(b_{1}, \ldots, b_{n}\right)-a .\left(p_{1}, \ldots, p_{n}\right)$ has non negative components and

$$
\forall X \in V_{2}, I^{\prime}(X)=E\left(Q_{X}(U) \cdot \phi(U)\right) .
$$

Proof. When the quantile operator $Q^{U}$ is the $\mu$-quantile of Galichon et al., we now from [6] that Axioms 1', 2', 3' and Inequality aversion is equivalent with the existence of a functional $I^{\prime}: V_{2} \rightarrow \mathbf{R}$ representing the same preference relation on $V_{2}$ as $I$, the existence of $a \in \mathbf{R}, b=\left(b_{1}, \ldots, b_{n}\right) \in \mathbf{R}^{n}$, such that $\phi\left(p_{1}, \ldots, p_{n}\right)=\left(b_{1}, \ldots, b_{n}\right)-$ a. $\left(p_{1}, \ldots, p_{n}\right)$ has non negative components and

$$
\forall X \in V_{2}, I^{\prime}(X)=E\left(Q_{X}(U) . \phi(U)\right) .
$$

which is equivalent to Theorem 3.3 (thus with the set of 6 assumptions on $I^{\prime}$ ).

\section{Unicity and elicitation of the parameters}

The following theorem shows that in our framework the parameters of the inequality mindedness evaluations are unique and depend on the policy maker evaluations of some specific distributions. In the following theorem, let $S=[0,1]^{n}$, $U\left(p_{1}, \ldots, p_{n}\right)=\left(p_{1}, \ldots, p_{n}\right), P$ be the Lebesgue measure on $[0,1]^{n}, Q_{X}$ be the the associated $U$-quantile operator when $U(p)=p$.

Theorem 5.1. Let $I: V_{2} \rightarrow \mathbf{R}$. Assume I satisfies Normalization, Monotonicity, Inequality aversion, Positive homogeneity and Additivity on Quantiles and constants. Assume the quantile operator $Q$ satisfies point (1) to (6) in Definition 3.1. Then

$$
\forall X \in V^{2}: I(X)=-a \int Q_{X}(p) \cdot p d P+b \int Q_{X}(p) d P,
$$

where

$$
a=\frac{\left.4-8 I\left(\mathbb{I}_{\left\{p_{1} \geq \frac{1}{2}\right\}}, \ldots, \mathbb{I}_{\left\{p_{n} \geq \frac{1}{2}\right\}}\right)\right)}{n}
$$

and

$$
b_{i}=\frac{a}{2}+I\left(0, \ldots, 0, \mathbb{1}_{S}, \ldots, 0\right) .
$$

Proof in Appendix 7.4 


\section{Discussion and concluding remarks}

As seen in corollay 3.6, choosing $U(p)=p$ allows to recover Yaari's theory for $n=1$. But the same choice $U(p)=p$ for $n>1$ may lead also to some controversial result, as illustrated below. Hereafter, we concentrate on the $\mu$-quantile of Galichon et al. The following result is proved in appendix 7.7.

Theorem 6.1. (most inegalitarian situation with fixed marginals) Let $U=\left(U_{1}, \ldots, U_{n}\right) \in$ $V_{2}$ whose probability law is absolutely continuous with respect to the Lebesgue measure. Under assumption of Theorem 4.1, for every $X \in V_{2}, \min _{X_{k}={ }_{d} Y_{k}, k=1, \ldots, n} I\left(Y_{1}, \ldots, Y_{n}\right)$ is reached at $Y=\left(Q_{X_{1}}^{U_{1}}\left(U_{1}\right), \ldots, Q_{X_{n}}^{U_{n}}\left(U_{n}\right)\right)$ where $Q_{X_{k}}^{U_{k}}$ denotes the $U_{k}$-quantile of $X_{k}$ for every $k=1, \ldots, n$.

Corollary 6.2. (most inegalitarian situation with fixed marginals when $U(p)=p$ ) If $U(p)=p$ then for every $Y$ in Theorem above, the components of $Y=\left(Y_{1}, \ldots, Y_{n}\right)$ are pairwise independent.

Proof. Indeed, the theorem above gives $Y=\left(Q_{X_{1}}^{U_{1}}\left(p_{1}\right), \ldots, Q_{X_{n}}^{U_{n}}\left(p_{n}\right)\right)$ whose components are clearly independent with respect to Lebesgue measure.

Corollary 6.3. There does not exist $U \in V_{2}$ whose probability law is absolutely continuous with respect to the Lebesgue measure such that for every $X \in V_{2}$,

$$
\min _{X_{k}={ }_{d} Y_{k}, k=1, \ldots, n} I\left(Y_{1}, \ldots, Y_{n}\right)
$$

is reached at a comonotonic vector.

Proof. By contradiction: if such $U$ exists, consider $X=U$. Since the $U_{k}$-quantile of $X_{k}$ is identity, the above theorem gives that $\min _{X_{k}={ }_{d} Y_{k}, k=1, \ldots, n} I\left(Y_{1}, \ldots, Y_{n}\right)$ is reached at $Y=U$. But if $U$ is comonotonic, the support $S$ of the probability measure $\mu$ of $U$ cannot contain any subset of dimension larger than 1. But then the Lebesgue measure of $S$ should be 0 , thus by absolute continuity, $\mu(S)=0$, a contradiction.

Intuitively the most inegalitarian situations should occur when the marginals are comonotonic. The above results show that the previous framework does not allow it. 
Substituting $S=[0,1]$ to $S=[0,1]^{n}$ and defining $I(X)$ as in Theorem 5.1, where $P$ is now the Lebesgue measure on $[0,1]$ and $U(p)=(p, \ldots, p)$, one can easily see that with this formulation, the most inegalitarian situation occurs when the marginals are comonotonic. Indeed, a similar argument to Theorem 6.1 gives that the most inegalitarian situations is reached at $Y=\left(F_{X_{1}}^{-1}, \ldots, F_{X_{n}}^{-1}\right)$ which is clearly comonotonic. It will be the purpose of a future research to axiomatize such formula, noticing that in such a case technical difficulties arise, because the law of $U$ is no longer absolutely continuous with respect to the Lebesgue measure.

\section{Appendix}

\subsection{Proof of Proposition 2.1}

(i) For every $X \in V_{2}$, the random variables $\tilde{Q}_{X}$ and $X$ have the same law (see [5], Lemma A19 p.408 ),

(ii) For every $X, Y \in V_{2}, Q_{X}+Q_{Y}$ is itself the quantile of some random variable in $V^{2}$ (for example the quantile of $\tilde{Q}_{X}+\tilde{Q}_{Y}$ ).

(iii) For every $\lambda \geq 0$ and $X$ in $V_{2}, Q_{\lambda X}$ is itself the quantile of some random variable in $V^{2}$ (for example the quantile of $\lambda \tilde{Q}_{X}$ ).

(iv) The quantile of every constant random variable $k$ on $(S, \mathcal{F}, P)$ is equal to itself (almost surely).

\subsubsection{Reminders of optimal transportation and optimal coupling.}

Consider hereafer a random vector $U \in V_{2}$. Sometimes, we shall assume that the law of $U$ is absolutely continuous with respect to Lebegue measure: this means that for every borelian subset $A \subset \mathbb{R}^{n}$ of Lebesgue-measure $0, P^{U}(A)=0$.

An important object hereafter is $\rho_{U}$, the maximal correlation functional with respect to $U$ : this is the real function defined on $V_{2}$ by

$$
(\mathcal{P}) \forall X \in V_{2}, \rho_{U}(X)=\sup _{\tilde{X}={ }_{d} X} E(\tilde{X} . U)
$$


From Optimal coupling theory, given $X \in V_{2}$, we have the following proposition:

Proposition 7.1. Assume $U$ is absolutely continuous with respect to Lebegue measure. Then:

(i) Existence and uniqueness: there exists a solution $\tilde{X} \in V_{2}$ of $(\mathcal{P})$, unique (almost everywhere). The pair $(\tilde{X}, U)$ is called an optimal coupling. ${ }^{2}$

(ii) Form of the solution: a solution $\tilde{X} \in V_{2}$ can be written $\tilde{X}=\nabla f(U)$ for $f$ : $\mathbf{R}^{n} \rightarrow \mathbf{R}$ convex and lower semicontinuous. Moreover, $\nabla f$ in the previous decomposition is unique, which means that if $\nabla f(U)=\nabla g(U)$ is a solution of $(\mathcal{P})$, where $f, g: \mathbf{R}^{n} \rightarrow \mathbf{R}$ are convex and l.s.c., then $\nabla f=\nabla g$.

(iii) Characterization of the solution: if $f: \mathbf{R}^{n} \rightarrow \mathbf{R}$ convex and l.s.c. function such that $\nabla f(U)={ }_{d} X$ then $\nabla f(U)$ is a solution of $(\mathcal{P})$.

(iv) Symmetry:

$$
\sup _{\tilde{X}==_{d} X, \tilde{U}={ }_{d} U} E(\tilde{X} \cdot \tilde{U})=\sup _{\tilde{X}=d X} E(\tilde{X} \cdot U)=\sup _{\tilde{U}={ }_{d} U} E(X \cdot \tilde{U}) .
$$

Remark 7.1. In particular, when $U$ is absolutely continuous with respect to Lebesgue measure, this proves the existence of a convex and l.s.c. function $f: \mathbf{R}^{n} \rightarrow \mathbf{R}$ such that $\nabla f$ exists almost surely, and $\nabla f(U)$ has the same law as $X$.

Now, the equality $\rho_{U}(X)=\sup _{\tilde{U}={ }_{d} U} E(X . \tilde{U})$ proves that $\rho_{U}$ depends only on the law of $U$. Thus, one could define equivalently, for every probability measure $\mu$ with a finite second moment:

$$
\rho_{\mu}(X)=\sup _{\tilde{X}={ }_{d} X} E(\tilde{X} \cdot U)
$$

where $U$ is any element in $V_{2}$ whose law is $\mu$. With the previous notations, we could note $\rho_{P U}=\rho_{U}$.

2. Obviously, $\tilde{X}$ depends on $U$, not only on the law of $U$. 


\subsection{Proof that Galichon et al. $\mu$-quantile defines a quantile operator}

We have to prove the 3 points of the definition of a quantile operator, but we will prove as a matter of fact that the 3 additional properties are also true (the last one in the particular case where $S=[0,1]^{n}, U\left(p_{1}, \ldots, p_{n}\right)=\left(p_{1}, \ldots, p_{n}\right)$ and $P$ is the Lebesgue measure.)

Point i) is true by definition of $\mu$-quantile.

For Point iii), remark that from optimal coupling theory (see reminders above)

$$
\sup _{\tilde{X}={ }_{d} X} E(\tilde{X} \cdot U)=\sup _{\tilde{U}={ }_{d} U} E(\tilde{U} \cdot X) .
$$

In particular, the $\mu$-quantile of $X+\lambda\left(\mathbb{I}_{S}, \ldots, \mathbb{I}_{S}\right)$ satisfies

$$
E\left(Q_{X+\lambda\left(\mathbb{I}_{S}, \ldots, \mathbb{I}_{S}\right)}(U) \cdot U\right)=\sup _{\tilde{U}={ }_{d} U} E\left(\tilde{U} \cdot\left(X+\lambda\left(\mathbb{I}_{S}, \ldots, \mathbb{I}_{S}\right)\right)=\sup _{\tilde{U}={ }_{d} U} E(\tilde{U} \cdot X)+\lambda E(U) .\right.
$$

which is also equal to

$$
\sup _{\tilde{X}={ }_{d} X} E(U \cdot \tilde{X})+\lambda E(U)=E\left(Q_{X}(U) \cdot U\right)+\lambda E(U)=E\left(\left(Q_{X}(U)+\lambda\left(\mathbb{I}_{S}, \ldots, \mathbb{I}_{S}\right)\right) \cdot U\right) .
$$

and since the law of $Q_{X}(U)+\lambda\left(\mathbb{I}_{S}, \ldots, \mathbb{I}_{S}\right)$ is the law of $X+\lambda\left(\mathbb{I}_{S}, \ldots, \mathbb{I}_{S}\right)$, we get that $Q_{X+\lambda\left(\mathbb{I}_{S}, \ldots, \mathbb{I}_{S}\right)}=Q_{X}+\lambda\left(\mathbb{I}_{S}, \ldots, \mathbb{I}_{S}\right)$.

Point iv) comes from the fact that $f(p) \rightarrow \frac{\sum_{k=1}^{n} \lambda_{k} p_{k}^{2}}{2}$ is continuous and convex, thus $\nabla f(p)=\left(\lambda_{1} p_{1}, \ldots, \lambda_{n} p_{n}\right)$ is the quantile function of $f(U)$. For Point ii), we use the following lemma:

Lemma 7.2. i) The $\mu$-quantile of $Q_{X}(U)+Q_{Y}(U)$ is $Q_{X}+Q_{Y}$.

ii) For every $\lambda \geq 0$, the $\mu$-quantile of $\lambda Q_{X}(U)$ is $\lambda . Q_{X}$.

Proof. i) Use the characterization of $\mu$-Quantile: considering any $U \in V_{2}$ such that $P^{U}=\mu, Q_{X}=\nabla f$ and $Q_{Y}=\nabla g$ for some convex and l.s.c. functions $f, g: \mathbf{R}^{n} \rightarrow$ $\mathbf{R}$, and $(\nabla f(U), U)$ and $(\nabla g(U), U)$ are optimal couplings (see Proposition 7.1, (ii)). Thus, from Proposition 7.1, (iii), $\left(Q_{X}+Q_{Y}\right)(U)=\nabla(f+g)(U)$ is a solution of

$$
\sup _{\tilde{X}={ }_{d} Q_{X}(U)+Q_{Y}(U)} E(\tilde{X} \cdot U)
$$


(because $f+g$ is convex and l.s.c., and $\nabla(f+g)(U)=Q_{X}(U)+Q_{Y}(U)$. Thus, by definition, $\nabla(f+g)=Q_{X}+Q_{Y}$ is the $\mu$-quantile of $Q_{X}(U)+Q_{Y}(U)$.

ii) Similar to i): let $\lambda \geq 0$. Then $Q_{X}=\nabla f$ for some convex and l.s.c. function $f: \mathbf{R}^{n} \rightarrow \mathbf{R}$, and $(\nabla f(U), U)$ is an optimal coupling. From Proposition 7.1, (iii), since $\lambda . f$ convex and l.s.c., $\lambda Q_{X}(U)=\nabla(\lambda f)(U)$ is a solution of

$$
\sup _{\tilde{X}={ }_{d} \lambda Q_{X}(U)} E(\tilde{X} \cdot U)
$$

Thus, by definition, $\nabla(\lambda f)=\lambda Q_{X}$ is the $\mu$-quantile of $\lambda Q_{X}(U)$.

Last, we prove the last point when $S=[0,1]^{n}, U\left(p_{1}, \ldots, p_{n}\right)=\left(p_{1}, \ldots, p_{n}\right)$ and $P$ is the Lebesgue measure. Thus, we want to show that if $X=\left(X_{1}, \ldots, X_{n}\right)$ where the $X_{i}$ 's are independent, then one has $Q_{X}(p)=\left(Q_{X_{1}}\left(p_{1}\right), \ldots, Q_{X_{i}}\left(p_{i}\right), \ldots, Q_{X_{n}}\left(p_{n}\right)\right)$ for every $p=\left(p_{1}, \ldots, p_{i}, \ldots, p_{n}\right) \in[0,1]^{n}$. From Optimal Coupling Theory, it is enough to prove that there exists $f: p \in[0,1]^{n} \longrightarrow f(p) \in \mathbf{R}$ such that $\mathrm{f}$ is convex, $\nabla f=Q_{X}$ and $Q_{X}={ }_{d} X$. Take $f\left(p_{1}, \ldots, p_{n}\right)=\sum_{i=1}^{n} \int_{0}^{p_{i}} Q_{X_{i}}(t) d t$, then $\mathrm{f}$ is convex and $\nabla f=Q_{X}$. Let us check that $Q_{X}={ }_{d} X . P\left(Q_{X_{1}} \leq x_{1}, \ldots, Q_{X_{i}} \leq x_{i}, \ldots, Q_{X_{n}} \leq x_{n}\right)=$ $\prod_{i=1}^{n} \nu\left(Q_{X_{i}}\left(p_{i}\right) \leq x_{i}\right)$ where $\nu$ the Lebesgue measure on $[0,1]$. Thus, $P\left(\ldots, Q_{X_{i}}\left(p_{i}\right) \leq\right.$ $\left.x_{i}, \ldots\right)=\prod_{i=1}^{n} F_{X_{i}}\left(x_{i}\right)$, hence $Q_{X}={ }_{d} X$.

\subsection{Proof of Proposition 3.4}

Proof. First, from its definition, $Q_{X}$ only depends on the distribution of $X$. Moreover, the proof of $Q_{X}(U)={ }_{d} X$ can be found in [8], page 14. Thus Point 1 in Definition 3.1 is satisfied. Second, we will prove that the $U$-quantile of $Q_{X}(U)+Q_{Y}(U)$ is $Q_{X}+Q_{Y}$ (where $X=\left(X_{1}, X_{2}\right)$ and $Y=\left(Y_{1}, y_{2}\right)$ are both 2-random variables), which will prove Point 2 in Definition 3.1 . First, by definition of $U$-quantile, the first component of the $U$-quantile of $Q_{X}(U)+Q_{Y}(U)$ is the standard one-dimensional quantile of $F_{X}^{-1}\left(U_{1}\right)+F_{Y}^{-1}\left(U_{1}\right)$, which is $F_{X}^{-1}+F_{Y}^{-1}$ (see Proposition 2.1). Now, we check the same result for the second component. This is essentially the same proof as for the first component, but with a conditionnal argument. Let $Z=Q_{X}(U)+Q_{Y}(U)$. By definition,

$$
Q_{Z}^{2}\left(p_{1}, p_{2}\right)=\inf \left\{x \in \mathbf{R}: P\left(Q_{X}^{2}(U)+Q_{Y}^{2}(U) \leq x \mid F_{Q_{X}^{1}+Q_{Y}^{1}}^{-1}\left(U_{1}\right)=F_{Q_{X}^{1}+Q_{Y}^{1}}^{-1}\left(p_{1}\right)\right) \geq p_{2}\right\}
$$


But as explained before, the one-dimensional quantile of $Q_{X}^{1}+Q_{Y}^{1}=F_{X_{1}}^{-1}\left(U_{1}\right)+$ $F_{Y_{1}}^{-1}\left(U_{1}\right)$ is $F_{X_{1}}^{-1}+F_{Y_{1}}^{-1}$, thus we get

$$
Q_{Z}^{2}\left(p_{1}, p_{2}\right)=\inf \left\{x \in \mathbf{R}: P\left(Q_{X}^{2}(U)+Q_{Y}^{2}(U) \leq x \mid E\right) \geq p_{2}\right\}
$$

where $E=\left\{s \in S: F_{X_{1}}^{-1}\left(U_{1}(s)\right)+F_{Y_{1}}^{-1}\left(U_{1}(s)\right)=F_{X_{1}}^{-1}\left(p_{1}\right)+F_{Y_{1}}^{-1}\left(p_{1}\right)\right\}$.

This can be written

$$
\left.Q_{Z}^{2}\left(p_{1}, p_{2}\right)=\inf \left\{x \in \mathbf{R}: P_{E}\left(Q_{X}^{2}(U)+Q_{Y}^{2}(U)\right) \leq x\right) \geq p_{2}\right\},
$$

where $P_{E}$ denotes $P$ conditionnally to the event $E$.

Thus $p_{1}$ being fixed, $Q_{Z}^{2}\left(p_{1},.\right)$ is the one-dimensional quantile of $Z=Q_{X}^{2}(U)+$ $Q_{Y}^{2}(U)$ in the new probability space $\left(S, \mathcal{F}, P_{E}\right)$. We shall use the following lemma to re-inforce this statement:

\section{Lemma 7.3.}

$$
\left.Q_{Z}^{2}\left(p_{1}, p_{2}\right)=\inf \left\{x \in \mathbf{R}: P_{E}\left(Q_{X}^{2}\left(p_{1}, U^{2}\right)+Q_{Y}^{2}\left(p_{1}, U^{2}\right)\right) \leq x\right) \geq p_{2}\right\} .
$$

We have to prove that the conditionnal probability allows to replace $U^{1}($.$) in the$ probability above by $p_{1}$. First, remark that $E=E 1 \cap E_{2}$ where $E_{1}=\{s \in S$ : $\left.\left.F_{X_{1}}^{-1}\left(U_{1}(s)\right)=F_{X_{1}}^{-1}\left(p_{1}\right)\right)\right\}$ and $\left.E_{2}=\left\{s \in S: F_{Y_{1}}^{-1}\left(U_{1}(s)\right)=F_{Y_{1}}^{-1}\left(p_{1}\right)\right)\right\}$, which is a consequence of the comonotonicity of $F_{X_{1}}^{-1}\left(U_{1}\right)$ and $F_{Y_{1}}^{-1}\left(U_{1}\right)$. Indeed, clearly, $E_{1} \cap E_{2} \subset$ $E$. To prove the other inclusion, let $\bar{s} \in S$ such that $p_{1}=U_{1}(\bar{s})$ (in particular $\bar{s} \in E$ ), and take $s^{\prime} \notin E_{1} \cap E_{2}$. In a first case (the other cases being treated similarly), we have $F_{X_{1}}^{-1}\left(U_{1}\right)\left(s^{\prime}\right)>F_{X_{1}}^{-1}\left(U_{1}(\bar{s})\right)$. Then by comonotonicity, $F_{Y_{1}}^{-1}\left(U_{1}\right)\left(s^{\prime}\right) \geq F_{Y_{1}}^{-1}\left(U_{1}(\bar{s})\right)$, thus, by summing these inequalities, we get $s^{\prime} \notin E$; the other cases are similar.

Thus, $P_{E}\left(Q_{X}^{2}\left(U^{1}, U^{2}\right)+Q_{Y}^{2}\left(U^{1}, U^{2}\right) \leq x\right)$ is equal to the probability of

$$
\begin{aligned}
& \left\{s \in S: \inf \left\{x^{\prime} \in \mathbf{R}: P\left(s^{\prime} \in S: X^{2}\left(s^{\prime}\right) \leq x^{\prime}\right) \geq U^{2}(s) \mid F_{X_{1}}^{-1}\left(U_{1}\left(s^{\prime}\right)\right)=F_{X_{1}}^{-1}\left(U^{1}(s)\right)\right)\right\} \\
& \left.+\inf \left\{x^{\prime} \in \mathbf{R}: P\left(s^{\prime} \in S: Y^{2}\left(s^{\prime}\right) \leq x^{\prime}\right) \geq U^{2}(s) \mid F_{Y_{1}}^{-1}\left(U_{1}\left(s^{\prime}\right)\right)=F_{Y_{1}}^{-1}\left(U^{1}(s)\right)\right\} \leq x\right\}
\end{aligned}
$$

conditionnaly to

$$
E=E_{1} \cup E_{2}=\left\{s \in S: F_{X_{1}}^{-1}\left(U_{1}(s)\right)=F_{X_{1}}^{-1}\left(p_{1}\right) \text { and } F_{Y_{1}}^{-1}\left(U_{1}(s)\right)=F_{Y_{1}}^{-1}\left(p_{1}\right)\right\} \text {, }
$$


which is thus the probability of

$$
\begin{aligned}
& \left\{s \in S: \inf \left\{x^{\prime} \in \mathbf{R}: P\left(s^{\prime} \in S: X^{2}\left(s^{\prime}\right) \leq x^{\prime}\right) \geq U^{2}(s) \mid F_{X_{1}}^{-1}\left(U_{1}\left(s^{\prime}\right)\right)=F_{X_{1}}^{-1}\left(p_{1}\right)\right)\right\} \\
& \left.\left.+\inf \left\{x^{\prime} \in \mathbf{R}: P\left(s^{\prime} \in S: Y^{2}\left(s^{\prime}\right) \leq x^{\prime}\right) \geq U^{2}(s) \mid F_{Y_{1}}^{-1}\left(U_{1}\left(s^{\prime}\right)\right)=F_{Y_{1}}^{-1}\left(p_{1}\right)\right)\right\} \leq x\right\}
\end{aligned}
$$

conditionnaly to $E$, which is exactly the conclusion of the lemma.

The previous lemma proves that $p_{1}$ being fixed, $Q_{Z}^{2}\left(p_{1},.\right)$ is the one-dimensional quantile of $Z=Q_{X}^{2}\left(p_{1}, U^{2}\right)+Q_{Y}^{2}\left(p_{2}, U^{2}\right)$ in the new probability space $\left(S, \mathcal{F}, P_{E}\right)$. Note that the law of $U^{2}$ with respect to the probability $P_{E}$ is absolutely continuous with respect to the Lebesgue measure on $\mathbf{R}$, simply because $U^{2}$ is independent from $E$. Thus, we know that $Q_{Z}^{2}\left(p_{1},.\right)$ is characterized by $Q_{Z}^{2}\left(p_{1},.\right)=\nabla g$ with $\nabla g(U)={ }_{d} Z$,

where $g:[0,1] \rightarrow \mathbf{R}$ is a convex function. But if we take $g(t)=\int_{0}^{t}\left(Q_{X}^{2}\left(p_{1}, u\right)+\right.$ $\left.Q_{Y}^{2}\left(p_{1}, u\right) d u\right)$, it satisfies $\nabla g(U)={ }_{d} Z$, thus we only have to prove that $\nabla g$ is increasing (thus $g$ convex).

But exactly as Lemma 7.3 above, we get that $Q_{X}^{2}\left(p_{1},.\right)$ (resp. $\left.Q_{Y}^{2}\left(p_{1},.\right)\right)$ is the one-dimensional quantile of $X$ (resp. of $Y)$ in $\left(S, \mathcal{F}, P_{E_{1}}\right)\left(\operatorname{resp}\right.$. in $\left.\left(S, \mathcal{F}, P_{E_{2}}\right)\right)$. In particular, $Q_{X}^{2}\left(p_{1},.\right)$ and $Q_{Y}^{2}$ are increasing, thus $\nabla g=Q_{X}^{2}\left(p_{1},.\right)+Q_{Y}^{2}\left(p_{1},.\right)$ is increasing. Finally, $Q_{Z}^{2}\left(p_{1}, p_{2}\right)=Q_{X}^{2}\left(p_{1}, p_{2}\right)+Q_{Y}^{2}\left(p_{1}, p_{2}\right)$.

We prove similarly that the $U$-quantile of $\lambda Q_{X}(U)$ is $\lambda Q_{X}$, which ends the proof.

\subsection{Proof of Theorem 3.3}

Step one: first prove that if the quantile operator $Q$ satisfies the basic assumptions (1), (2) and (3), and if I satisfies Assumptions (1)-(6), then there exists $\phi:[0,1]^{n} \rightarrow \mathbf{R}^{n}$, with non negative components, such that (i) $E\left(\left(\mathbb{1}_{S}, \ldots, \mathbb{1}_{S}\right) \cdot \phi(U)\right)=1$, (ii) for every $X \in V_{2}^{\prime}, I(X)=\int Q_{X}(U) \cdot \phi(U) d P=\min _{\tilde{X}={ }_{d} X} \int \tilde{X} \cdot \phi(U) d P$.

Define

$$
W=\left\{X:\left([0,1]^{n}, \mathcal{B}_{[0,1]^{n}}, P^{U}\right) \rightarrow\left(\mathbb{R}^{n}, \mathcal{B}_{\mathbb{R}^{n}}\right) \text { measurable }\right\}
$$

where $P^{U}$ is the probability measure of $U$ on $[0,1]^{n}$, and let $W_{2}$ be the set of squareintegrable random variables of $W$. Remark that in the particular case where $U(p)=p$ and $P$ is the Lebesgue measure, then $W_{2}=V_{2}$. 
Define

$$
C=\left\{Q_{X}: X \in V_{2}^{\prime}\right\}
$$

the subset of $W_{2}$ whose elements are all possible quantiles of random variables in $V_{2}^{\prime}$. From the definition of quantiles, $C$ is a cone ${ }^{3}$. Indeed, if $\lambda \geq 0$, then for every $X \in V_{2}^{\prime}$, $\lambda Q_{X}$ is the quantile of some $X^{\prime} \in V_{2}^{\prime}$ (Point 2 of Quantile operator definition), thus $\lambda Q_{X} \in C$. Similarly, if $X, Y$ are in $V_{2}^{\prime}$, then $Q_{X}+Q_{Y}$ is a quantile of some $Z \in V_{2}^{\prime}$. Last, $0 \in C$ since 0 is the quantile of itself.

Let $F \subset W_{2}$ be the vector space spanned by $C$. It can be written

$$
F=C-C=\left\{c-c^{\prime}:\left(c, c^{\prime}\right) \in C \times C\right\}
$$

Now, define $\tilde{I}: F \rightarrow \mathbb{R}$ by

$$
\forall(X, Y) \in V_{2}^{\prime} \times V_{2}^{\prime}: \tilde{I}\left(Q_{X}-Q_{Y}\right)=I\left(Q_{X}(U)\right)-I\left(Q_{Y}(U)\right)
$$

Since $I$ satisfies Positive Linearity on Quantiles, $\tilde{I}$ is linear on $F$. Let $p: W_{2} \rightarrow \mathbb{R}$ be defined by

$$
\forall X \in W_{2}, p(X)=-I(-X(U))
$$

We have the following properties:

- First, $W_{2}$ and $F$ are Riesz spaces (i.e. partially ordered vector spaces which also are lattices) for the natural order defined by $X \lesssim Y \Leftrightarrow X(s) \leq Y(s)$ for almost every $s \in S$.

- Second, the function $p$ satisfies Monotonicity (because $I$ satisfies Monotonicity), i.e., $X \leq Y \Rightarrow p(X) \leq p(Y)$.

— Third, $p$ is a sublinear function, which means the following properties (1) and (2) are true:

(1) we have $p(X+Y) \leq p(X)+p(Y)$ for every $X$ and $Y$ in $W_{2}$.

(2) $p$ is positively homogeneous.

Property (1) is true from Inequality Aversion Assumption (see Point 3 in Remark 3.2). Property (2) is true because $I$ is itself positively homogeneous.

- Fourth, $\tilde{I}$ is positive on $F$, that is $Q_{X}-Q_{Y} \geq 0$ implies $\tilde{I}\left(Q_{X}-Q_{Y}\right) \geq 0$ from Monotonicity of $I$.

3. Throughout this paper, cone will be used for convex cone containing 0 . 
- Last, we have $\tilde{I}\left(Q_{X}-Q_{Y}\right) \leq p\left(Q_{X}-Q_{Y}\right)$ for all $Q_{X}, Q_{Y} \in C$. Indeed, From Inequality aversion, $\left.I\left(Q_{X}(U)\right)+I\left(\left(-Q_{X}(U)\right)+Q_{Y}(U)\right)\right) \leq I\left(Q_{X}(U)+\right.$ $\left.Q_{Y}(U)+\left(-Q_{X}(U)\right)\right)=I\left(Q_{Y}(U)\right)$, thus $\tilde{I}\left(Q_{X}-Q_{Y}\right)=I\left(Q_{X}(U)\right)-I\left(Q_{Y}(U)\right) \leq$ $\left.-I\left(\left(-Q_{X}(U)\right)+Q_{Y}(U)\right)\right)=p\left(Q_{X}-Q_{Y}\right)$.

By Hahn-Banach Extension Theorem (Th. 8.31 in [1]), and since from above $\tilde{I}$ is a positive linear function on $F$, majorized by the monotone sublinear function $p, \tilde{I}$ extends to a linear functional $\bar{I}$ on $W_{2}$, satifying

$$
\bar{I}(X) \leq p(X) \forall X \in W_{2} .
$$

Moreover, if $X \geq 0$, then

$$
\bar{I}(-X)=-\bar{I}(X) \leq p(-X) \leq p(0)=0
$$

from positive homogeneity and Monotonicity of $p$. This proves that the extension $\bar{I}$ is a positive operator.

Recall in the following that a norm $\|\cdot\|$ on $W_{2}$ is a lattice norm if $|X| \leq|Y|$ a.e. $\Rightarrow$ $\|X\| \leq\|Y\|$. In particular, this is the case for $\|\cdot\|_{2}$. A Banach lattice is, by definition, a Banach space for the lattice norm. Again, this is true for $\left(W_{2},\|\cdot\|_{2}\right)$, which is even a Hilbert space.

Since every positive operator on a Banach lattice is continuous (see Theorem 9.6, p. 350 in [1]), $\bar{I}$ is continuous on $W_{2}$, which is a Hilbert space for the scalar product $<X, Y>=\int X . Y d P$. Thus, from Riesz representation theorem, there is $\phi \in W_{2}$ with non negative components on the support of $P^{U}$ (because $\bar{I}$ is positive) such that

$$
\forall X \in W_{2}, \bar{I}(X)=\int_{[0,1]^{n}} X(p) \phi(p) d P^{U}
$$

In particular, taking any $X \in V_{2}^{\prime}$, we get

$$
I(X)=I\left(Q_{X}(U)\right)=\bar{I}\left(Q_{X}\right)=\int_{[0,1]^{n}} Q_{X}(p) \cdot \phi(p) d P^{U}=\int_{S} Q_{X}(U) \cdot \phi(U) d P
$$

Note that

$$
\int\left(\mathbb{I}_{S}, \ldots, \mathbb{I}_{S}\right) \cdot \phi(U) d P=\int_{[0,1]^{n}}(1, \ldots, 1) \cdot \phi d P^{U}=I\left(\mathbb{I}_{S}, \ldots, \mathbb{I}_{S}\right)=1
$$

because the Quantile of $\left(\mathbb{I}_{S}, \ldots, \mathbb{I}_{S}\right)$ is $(1, \ldots, 1)$. 
Now, for every $Y \in W_{2}$ such that $X(U)={ }_{d} Y(U)$,

$$
\bar{I}(-Y)=\int-Y(U(p)) \cdot \phi(U(p)) d P \leq p(-Y)=-I(Y(U))=-I(X(U))
$$

where

$$
-I(X(U))=-I\left(Q_{X(U)}(U)\right)=-\tilde{I}\left(Q_{X(U)}\right)=-\bar{I}\left(Q_{X(U)}\right)=-\int Q_{X(U)}(U) \cdot \phi(U) d P
$$

that is

$$
\forall Y \in W_{2} \text { such that } Y(U)={ }_{d} X(U), \int Y(U) \cdot \phi(U) d P \geq \int Q_{X(U)}(U) \cdot \phi(U) d P
$$

Now given $X^{\prime} \in V_{2}$, there exists $X \in W_{2}$ such that $X(U)={ }_{d} X^{\prime}$ (from optimal coupling theory, because $U$ is absolutely continuous with respect to Lebesgue measure).

Thus the previous equation gives

$$
\forall Y \in W_{2} \text { such that } Y(U)={ }_{d} X(U), \int Y(U) . \phi(U) d P \geq \int Q_{X^{\prime}}(U) . \phi(U) d P
$$

But every $Y^{\prime} \in V_{2}$ such that $Y^{\prime}={ }_{d} X^{\prime}$ can be written in a similar way $Y^{\prime}=Y(U)$ for some $Y \in W_{2}$, and finally we get

$$
\forall Y^{\prime} \in V_{2} \text { such that } Y^{\prime}={ }_{d} X^{\prime}, \int Y^{\prime} . \phi(U) d P \geq \int Q_{X^{\prime}}(U) . \phi(U) d P
$$

whis ends the proof of (ii).

Step two: Converse implication: Assume there exists $\phi:[0,1]^{n} \rightarrow \mathbf{R}^{n}$, with non negative components, such that (i) $E\left(\left(\mathbb{1}_{S}, \ldots, \mathbb{1}_{S}\right) . \phi(U)\right)=1$, (ii) for every $X \in V_{2}^{\prime}$,

$I(X)=\int Q_{X}(U) \cdot \phi(U) d P=\min _{\tilde{X}=_{d} X} \int \tilde{X} \cdot \phi(U) d P$, and let us prove I satisfies Assumptions (1)-(5).

Assumption (1) is exactly (i) above. Monotonicity, concavity, positive homogeneity and neutrality are a consequence of $I(X)=\min _{\tilde{X}={ }_{d} X} \int \tilde{X} . \phi(U) d P$, i.e. $I$ is the min correlation risk measure with respect to $\phi(U)$ (see remark 3.5). Additivity on quantiles is straighforward.

\subsection{Proof of Corollary 3.4}

Step one: Let us now assume the quantile operator satisfies additionally (4) in Definition 3.1 and that the support of $P^{U}$ is $[0,1]^{n}$, and let us prove that $\phi(p)=$ 
$\left(\phi\left(p_{1}\right), \ldots, \phi\left(p_{n}\right)\right)$.

Consider a diagonal $n$-matrix $D$ with strictly positive diagonal. From Point $(i v)$ in Definition 3.1, $p \rightarrow D p$ is the quantile of some $X^{D}(U) \in V_{2}^{\prime}$ for some $X^{D} \in W_{2}$, that is $D p=Q_{X^{D}(U)}(p)$, thus $D U(p)=Q_{X^{D}(U)}(U(p))$ whose law is the law of $X^{D}(U)$. Moreover, the previous equation implies that for every $Y \in W_{2}$ whose law is equal to the law of $X^{D}$

$$
\int Y(U) \cdot \phi(U(p)) d P \geq \int D U(p) \cdot \phi(U(p)) d P
$$

From optimal coupling theory, and since the law of $D U(p)$ is absolutely continuous with respect to the Lebesgue measure, there is some l.s.c. convex function $f^{D}: \mathbf{R}^{n} \rightarrow$ $\mathbf{R}$ such that $-\phi(U(p))=\nabla f^{D}(D U(p))$. Thus, $-\phi(x)=\nabla f^{D}(D x)$ for every $x \in[0,1]^{n}$. From Aleksandrof theorem, since $f^{D}$ is convex, $\nabla f^{D}$, thus $\phi$, are almost surely $C^{1}$ on ] $0,1\left[{ }^{n}\right.$.

Differentiating this equality at any $x \in] 0,1\left[^{n}\right.$, we get

$$
\nabla \phi(x)=-\nabla^{2} f^{D}(D x) \cdot D
$$

for every diagonal $n$-matrix $D$ with strictly positive diagonal, where $\nabla \phi(x)$ and $\nabla^{2} f(x)$ are assimilated to $n$-matrices.

Then, we use the following lemma:

Lemma 7.4. Let $A$ be a n-matrix.

i) Assume for every diagonal $n$-matrix $D$ with strictly positive diagonal, there exists a symmetric non negative matrix $B$ such that $A=-B D$. Then $A$ is diagonal with a negative diagonal.

ii) Moreover, if for every symmetric definite positive matrix $D$, there exists a symmetric non negative matrix $B$ such that $A=-B D$. Then $A=\lambda I_{n}$ for some negative $\lambda$.

Proof. Taking $D=I_{n}$ we get that $-A$ is symmetric non negative. Thus, $B$ and $D$ commutes, thus taking a diagonal matrix $D$ with distinct elements in the diagonal, we get $B$ diagonal, thus $A$ diagonal, and since $-A$ is symmetric non negative, $A$ has a 
negative diagonal. Then point ii) is straightforward.

From the lemma, $\nabla \phi(x)=\left(\nabla \phi_{1}(x), \ldots, \nabla \phi_{n}(x)\right)$ is diagonal for almost every $x$ in ] $0,1\left[n\right.$, with a negative diagonal. Thus $\phi(x)=\left(\phi_{1}\left(x_{1}\right), \ldots, \phi_{n}\left(x_{n}\right)\right)$ where each $\phi_{i}$ is decreasing.

Step two. Let now assume the quantile operator satisfies additionally (5) with $n \geq 2$, and let us prove that $\phi(p)=b-a p$.

Now, for every definite positive symmetric $n$-matrix $S$, from Point (5) of quantile operator definition, it turns out that $p \rightarrow S p$ is the quantile of some $X^{S}(U) \in V_{2}^{\prime}$, that is $S p=Q_{X^{S}(U)}(p)$. As above, from optimal coupling theory, and since the law of $Q_{X^{S}(U)}$ is absolutely continuous with respect to the Lebesgue measure, there is some l.s.c. convex function $f^{S}: \mathbf{R}^{n} \rightarrow \mathbf{R}$ such that $-\phi(U(p))=\nabla f^{S}(S U(p))$, thus $-\phi(x)=$ $\nabla f^{S}(S x)$ at every $x \in[0,1]^{n}$. Differentiating this equality at every interior point $x$, we get $-\nabla \phi(x)=\nabla^{2} f^{S}(S x) . S$ for every definite positive symmetric $n$-matrix $S$. From point ii) of Lemma above, $\nabla \phi(x)=\left(\nabla \phi_{1}(x), \ldots, \nabla \phi_{n}(x)\right)=\left(\lambda_{1}\left(x_{1}\right), \ldots, \lambda_{n}\left(x_{n}\right)\right)$ with $\lambda\left(x_{1}\right)=\ldots=\lambda_{n}\left(x_{n}\right) \leq 0$, thus $\nabla \phi(x)=-a(1, \ldots, 1)$ for some constant $a \geq 0$, and finally $\phi(x)=b-a x$ for some $b=\left(b_{1}, \ldots, b_{n}\right)$.

\subsection{Proof of Theorem 5.1}

From Theorem 2 and from Property (6) of quantile, when the $X_{1}, \ldots, X_{n}$ are mutually independent we get $I(X)=\sum_{i=1}^{n} \int_{0}^{1} F_{X_{i}}^{-1}(t)\left(b_{i}-a t\right) d t$.

It is immediate that $Q_{\left(0, \ldots, 0, S^{*}, 0, \ldots, 0\right)}=\left(0, \ldots, 0, S^{*}, 0, \ldots, 0\right)$ since $Q_{k}=k$.

If $\mathrm{k}$ is a constant hence denoting $\alpha_{i}=I\left(0, \ldots, 0, S^{*}, 0, \ldots, 0\right)$ one gets $\alpha_{i}=b_{i}-\frac{a}{2}$. (Note that from 1.2 of Theorem 2, $\alpha_{i}>0$. Furthermore, since $Q_{\left(S^{*}, \ldots, S^{*}\right)=\left(S^{*}, \ldots, S^{*}\right)}$ one gets $1=I\left(S^{*}, \ldots, S^{*}\right)=\sum_{i=1}^{n} \alpha_{i}$.

Let $f_{i}: t \in[0,1] \longrightarrow f_{i}(t) \in[0,1]$ be defined by:

$$
f_{i}(t)=\frac{1}{2 b_{i}-a}\left(a t^{2}+2\left(b_{i}-a\right) t\right) \text {, then } f_{i}(0)=0, f_{i}(1)=1, f_{i} \text { is increasing since } a>0
$$


and $\phi \geq 0$ implies $b_{i} \geq a, f_{i}$ is convex and $f_{i}^{\prime}(1-t)=\frac{b_{i}-a t}{\alpha_{i}}$. Therefore,

$$
I(X)=\sum_{i=1}^{n} \int_{0}^{1} F_{X_{i}}^{-1}(t) f_{i}^{\prime}(1-t) d t
$$

Note that $\int_{0}^{1} F_{X_{i}}^{-1}(t) f_{i}^{\prime}(1-t) d t$ is the measure of welfare allocated to attribute i when the policy maker applies the inequality distortion evaluation $f_{i}$, therefore $I(X)$ can be interpreted as the weighted average of the various attribute evaluations through the weights $\alpha_{i}$ allocated to each attribute $\mathrm{i}$ by the policymaker.

Finally notice that the derivation hence the unicity of a and b will be performed as soon as we can determine a. Actually since $\alpha_{i}=I\left(0, \ldots, 0, S^{*}, 0, \ldots, 0\right)$, we will obtain $b_{i}=\alpha_{i}+\frac{a}{2}$.

The next step aims at computing a.

Consider the events $A_{i}=\left\{p=\left(p_{1}, \ldots, p_{i}, \ldots, p_{n}\right) \in[0,1]^{n}, p_{i} \geq \frac{1}{2}\right\}$. Assume that these events are independent (which will be proved below) then applying the formula above for $X=\left(A_{1}^{*}, \ldots, A_{i}^{*}, \ldots, A_{n}^{*}\right)$, one gets $I(X)=\sum_{i=1}^{n} \int_{0}^{1} F_{A_{i}^{*}}^{-1}(t)\left(b_{i}-a t\right) d t$

Note that

$$
F_{A_{i}^{*}}^{-1}(t)=\left\{\begin{array}{l}
1, \text { if } \frac{1}{2} \leq t \leq 0 \\
0, \text { if } 0 \leq t \leq \frac{1}{2}
\end{array}\right.
$$

Taking into account that $b_{i}=\alpha_{i}+\frac{a}{2}$ and $\sum_{i=1}^{n} \alpha_{i}=1$, one gets that $I\left(A_{1}^{*}, \ldots, A_{i}^{*}, \ldots, A_{n}^{*}\right)=$ $\frac{1}{2}-\frac{n a}{8}$.

Hence letting $\alpha_{i}=I\left(0, \ldots, 0, S^{*}, 0, \ldots, 0\right), \beta=I\left(A_{1}^{*}, \ldots, A_{i}^{*}, \ldots, A_{n}^{*}\right)$, one gets that $a=\frac{4-8 \beta}{n}$ and $b_{i}=\alpha_{i}+\frac{a}{2}, \forall i=1, \ldots, n$.

It remains to prove that the $A_{i}$ 's are independent. This is immediate since:

$$
\begin{aligned}
& P\left(p=\left(p_{1}, \ldots, p_{i}, \ldots, p_{n}\right) \in[0,1]^{n}, p_{1} \geq \frac{1}{2}, \ldots, p_{n} \geq \frac{1}{2}\right)=\left(\frac{1}{2}\right)^{n}=\prod_{i=1}^{n} P(p= \\
& \left.\left(p_{1}, \ldots, p_{i}, \ldots, p_{n}\right) \in[0,1]^{n}, p_{i} \geq \frac{1}{2}\right)
\end{aligned}
$$

\subsection{Proof of Theorem 6.1}

Proof. From Theorem 4.1, we have

$$
I(Y)=E\left(Q_{Y}^{U}(U) \cdot\left(\left(b_{1}, \ldots, b_{n}\right)-a \cdot\left(U_{1}, \ldots U_{n}\right)\right)=\sum_{i=1}^{n} b_{i} E\left(Q_{Y}^{U}(U)_{i}\right)-a E\left(Q_{Y}^{U}(U) \cdot U\right) .\right.
$$


The first part is constant, because the law of $Q_{Y}^{U}(U)$ is equal to the law of $Y$, thus each component $Q_{Y}^{U}(U)_{i}$ has the same law as $X_{i}$. Consequently

$$
\min _{X_{k}={ }_{d} Y_{k}, k=1, \ldots, n} I\left(Y_{1}, \ldots, Y_{n}\right)=\sum_{i=1}^{n} b_{i} E\left(X_{i}\right)-a_{X_{k}={ }_{d} Y_{k}, k=1, \ldots, n} E\left(Q_{Y}^{U}(U) . U\right) .
$$

But

$$
\left.\max _{X_{k}={ }_{d} Y_{k}, k=1, \ldots, n} E\left(Q_{Y}^{U}(U) \cdot U\right)\right)=\max _{X_{k}={ }_{d} Y_{k}, k=1, \ldots, n} \sum_{i=1}^{n} E\left(Q_{Y}^{U}(U)_{i} \cdot U_{i}\right) .
$$

Since $Q_{Y}(U)$ has the same law as $Y$, it has the same marginals, thus $Q_{Y}(U)_{i}={ }_{d} X_{i}$ for every $i=1, \ldots, n$.

Thus

$$
\left.\max _{X_{k}={ }_{d} Y_{k}, k=1, \ldots, n} \sum_{i=1}^{n} E\left(Q_{Y}^{U}(U)_{i} \cdot U_{i}\right)\right) \leq \sum_{i=1}^{n} \max _{X_{i}={ }_{d} Y_{i}} E\left(Y_{i} \cdot U_{i}\right) .
$$

But from Optimal coupling theory, since each $U_{i}$ has a probability law on $\mathbf{R}$ which is absolutely continuous with respect to the Lebesgue measure, for every $i=1, \ldots, n$ we get

$$
\max _{X_{i}=d_{d} Y_{i}} E\left(Y_{i} \cdot U_{i}\right)=E\left(Q_{X_{i}}^{U_{i}}\left(U_{i}\right) \cdot U_{i}\right)
$$

where $Q_{X_{i}}^{U_{i}}=g_{i}^{\prime}$, almost surely, for some l.s.c. convex function $g_{i}$ on $U_{i}(S)$, and with $Q_{X_{i}}^{U_{i}}={ }_{d} X_{i}$

To finish, let us prove that $Y=\tilde{Y}:=\left(Q_{X_{1}}^{U_{1}}\left(U^{1}\right), \ldots, Q_{X_{n}}^{U_{n}}\left(U_{n}\right)\right)$ is a solution of

$$
\left.\max _{X_{k}={ }_{d} Y_{k}, k=1, \ldots, n} E\left(Q_{Y}^{U}(U) \cdot U\right)\right)
$$

From above, we only have to prove that $\tilde{Y}:=\left(Q_{X_{1}}^{U_{1}}\left(U^{1}\right), \ldots, Q_{X_{n}}^{U_{n}}\left(U_{n}\right)\right)$ can be written $Q_{Y}^{U}(U)$ for some $Y \in V_{2}$ with $X_{k}={ }_{d} Y_{k}$ for every $k=1, \ldots, n$.

From optimal coupling theory, given $Y, Q_{Y}^{U}$ is characterized as $Q_{Y}^{U}(U)=\nabla f(U)$ for some convex l.s.c. function $f: \mathbf{R}^{n} \rightarrow \mathbf{R}$ with $\nabla f(U)={ }_{d} Y$ (such $\nabla f$ is unique almost surely). Then define $Y=\tilde{Y}$ and $f\left(x_{1}, \ldots, x_{n}\right)=\left(g_{1}\left(x_{1}\right), \ldots, g_{n}\left(x_{n}\right)\right)$. Since each $g_{k}$ is l.s.c. convex on $U_{k}(S), f$ is l.s.c. and convex. Moreover, $\nabla f(U)=\tilde{Y}$, and from the previous characterization, $\tilde{Y}=Y=Q_{Y}^{U}(U)$, with $X_{k}={ }_{d} \tilde{Y}_{k}={ }_{d} Y_{k}, k=1, \ldots, n$. this ends the proof. 


\section{References}

[1] C. D. Aliprantis and K. C. Border. Infinite Dimensional Analysis. Springer, Berlin, 1994.

[2] G. Carlier, R. A. Dana, and A. Galichon. Pareto efficiency for the concave order and multivariate comonotonicity. Journal of Economic Theory, 147:207-229, 2012.

[3] Jan Dhaene, Michel Denuit, Marc J Goovaerts, Rob Kaas, and David Vyncke. The concept of comonotonicity in actuarial science and finance: theory. Insurance: Mathematics and Economics, 31(1):3-33, 2002.

[4] Tapani Lehtonen Elja Arjas. Approximating many server queues by means of single server queues. Mathematics of Operations Research, 3(3):205-223, 1978.

[5] H. Föllmer and A. Schied. Stochastic Finance: An Introduction in Discrete Time. De Gruyter studies in mathematics. Walter de Gruyter, 2004.

[6] A. Galichon and M. Henry. Dual theory of choice with multivariate risks. Journal of Economic Theory, 147:1501-1516, 2012.

[7] G. L. O'Brien. The comparison method for stochastic processes. The Annals of Probability, 3(1):80-88, 1975.

[8] L. Rüschendorf. Mathematical Risk Analysis: Dependence, Risk Bounds, Optimal Allocations and Portfolios. Springer, 2004.

[9] Ludger Ruschendorf. Ordering of distributions and rearrangement of functions. Ann. Probab., 9(2):276-283, 041981. 